\title{
Rule-based Activity Recognition Framework: Challenges, Technique and Learning
}

\author{
Holger Storf, Martin Becker \\ Fraunhofer IESE \\ Kaiserslautern, Germany \\ firstname.lastname@iese.fraunhofer.de
}

\author{
Martin Riedl \\ Hochschule Mannheim \\ Mannheim, Germany \\ Ma_Riedl@yahoo.de
}

\begin{abstract}
Among the central challenges of Ambient Assisted Living systems are the autonomous and reliable recognition of the assisted person's current situation and the proactive offering and rendering of adequate assistance services. In the context of emergency support, such situations may be acute emergency situations or long-term deviations from typical behavior that will result in emergency situations in the future. To optimize the treatment of the former and the prevention of the latter, reliable recognition of characteristic activities of daily living is necessary. In this paper, we present our multi-agent-based activity recognition framework as well as experiences made with it. Besides a detailed discussion of our hybrid recognition approach, we also elaborate on the tailoring of the underlying reasoning models to the individual environments and users in an initial learning phase. Finally, we present experiences made with the recognition framework in our Ambient Assisted Living Laboratory.
\end{abstract}

Activity Recognition; Ambient Assisted Living; Data Mining

\section{INTRODUCTION}

Driven by demographical and societal changes in most industrialized countries, the development of Ambient Assisted Living (AAL) systems seems to be a promising answer to the question of how to enable elderly or disabled people to live longer independent lives in their familiar residential environments [1]. Typical services comprise assistance in daily routine and, with the rising probability of emergencies, also emergency detection and prevention support. In order to render these services in a sensitive and proactive way and to protect the assisted persons from functional overload, these systems require a thorough understanding of the current context, e.g., location, current and past activities, and the assisted person's state of health.

The EMERGE project [2] aims at investigating situation recognition approaches that enable dependable emergency assistance services based upon unobtrusive sensors seamlessly integrated into the daily living environment. Therefore, the solution does not rely on cameras, microphones, and bodymounted tags, for instance. By monitoring the behavior patterns of assisted persons, the system automatically reasons on deviations in behavior and assesses the functional health status of the assisted person. If suspicious situations or upcoming trends are detected, the system provides stepwise adequate assistance to the assisted person by checking with the user and notifying external helpers, e.g. a socio-medical service center, who handle further preventive measures or rescue missions.
There are various well-known approaches for monitoring a person's movement and state of health [3]. However, recognizing activities, esp. complex ones such as Activities of Daily Living (ADL) [4] is, for various reasons, still a challenging issue. There are some promising approaches for fulfilling special requirements, but there is no known one-sizefits-all solution corresponding to the given problems.

In this paper, we present our multi-agent-based activity recognition framework and experiences made with it. Besides a detailed discussion of our hybrid recognition approach, we also elaborate on the tailoring of the underlying reasoning models to the individual environments and users in an initial learning phase. Finally, we present experiences made with the recognition framework in our Ambient Assisted Living Environment [5]. The paper is structured as follows: In section II, we introduce the AAL domain. The general problem of activity recognition is described in section III. Section IV discusses our multi-agent-based approach for solving this problem. In section $\mathrm{V}$, the realization of the approach is explained with a short introduction to the defined rule format and the automated learning algorithm. The paper closes with a conclusion and gives an outlook on planned future work.

\section{AAL DOMAIN AND EMERGE}

Ambient Assisted Living (AAL) [1] denotes concepts, products, and services that interlink and improve new technologies and social systems, with the aim of enhancing the quality of life for all people during all stages of their lives. AAL could therefore be translated best as intelligent systems of assistance for a better and safer life [6]. The potential range of services belonging to the AAL domain is huge. It encompasses any assistive service that facilitates daily life. The classification scheme [7] shown in Figure 1 structures this domain into six stereotypical subdomains with clearly separated responsibilities.

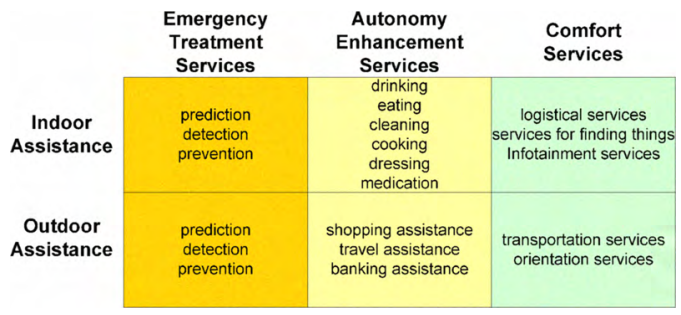

Figure 1. A classification scheme for the AAL services [7] 
Emergency treatment services aim at the early prediction of and recovery from critical conditions that might result in an emergency situation, and the safe detection and alert propagation of emergency situations. Autonomy enhancement services enable independent living for the assisted person who lacks certain capabilities. Comfort services cover all areas that do not fall into the former categories. These services facilitate daily life, but are not necessarily required. The majority of these assistance services render their service in a proactive and sensitive way to relieve the end users from cognitive loads. In order to do so, the services require a thorough understanding of the current and past activities and situations of the assisted person and their environment.

In the context of automated emergency treatment, for instance, short-term situations comprise automatic, reliable, and immediate detection of emergency situations, such as sudden falls or helplessness, or long-term deviations from typical behavior and vital parameters. Another type of critical situations from the medical point of view are special incidences of long-term trends, which are indicators of mental changes, e.g., dementia or depression, or other age-related diseases suffered by elderly people. Based on the recognized situation and health condition, adequate assistance services can be rendered in a proactive and preventive way [8]. In addition to that, the rate of false alarms could be reduced if knowledge about the current situation were available. Figure 2 provides an overview of the situations to be recognized within the EMERGE system.

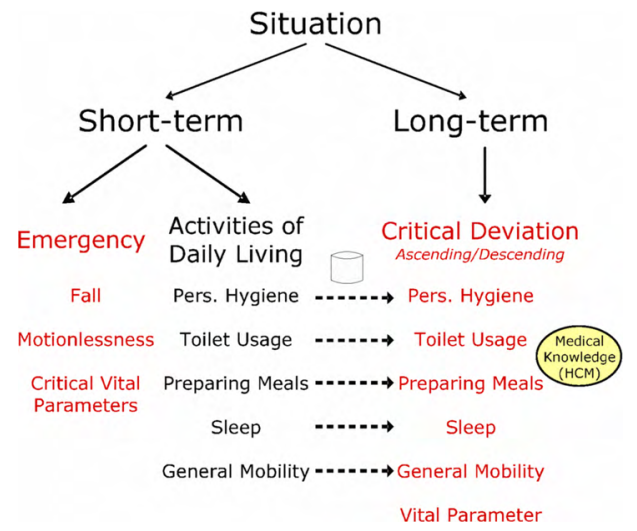

Figure 2. Situations in EMERGE

Of special interest in behavioral monitoring and health state assessment are the so-called Activities of Daily Living (ADLs), which were defined for the first time in the Katz index [9]. Based on ADLs, long-term trend analyses can be made and checked against the normal behavior and additional clinical information of the assisted person. Thus, deviations or inconsistencies provide indicators for the assisted person's health status and proactive assistance can be initialized if needed.

While trying to automate ADL recognition [10] and assessment based on environmental data in the EMERGE project, we encountered some challenges that will be discussed in the following section.

\section{ACTIVITY RECOGNITION - PROBLEMS AND APPROACHES}

A central challenge faced by Ambient Assisted Living systems is the question of which reasoning approach to follow for detecting complex human activities such as ADLs based upon a continuous sequence of events (states and state changes) measured by the different sensing systems. This becomes even more complicated if the sensors are integrated into the environment and thus cannot be directly correlated with a user. Figure 3 illustrates these challenges in an abstract way.

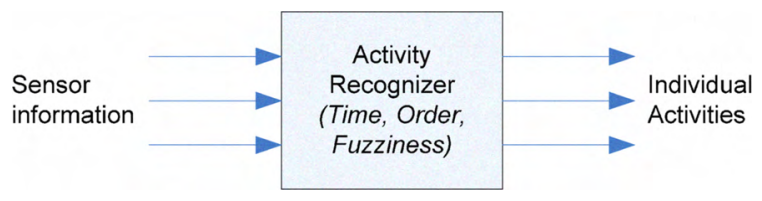

Figure 3. Problem of activity recognition in AAL

Obvious quality demands have to be considered, e.g. timeliness recognition and reliability in terms of precision and recall. One important challenge that complicates the selection of appropriate approaches is that the sensor configuration information typically varies in every system instance, for instance, depending on the layout of apartment, the applied sensor and communication devices, and the type of sensor data provided to the activity recognizer such as. raw, fused or already aggregated data. Furthermore, recognition is complicated by the individual behavior of people resulting in varying event or activity sequences. On the one hand, the typical activity sequence may show a clearly identifiable temporal order (e.g., activity 1 in Figure 4). On the other hand, these sequences can also be seen as almost randomly received sensor information (activity 2 ).

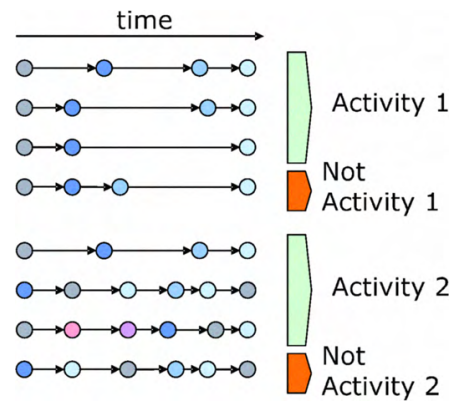

Figure 4. Example of event sequences

Examples of these cases may be the ADLs Toilet Usage and Preparation of Meals. In general, the usage of the toilet is definable as a clearly structured sequential chain. Contrary to this, the preparation of a meal has very high diversity, because the concrete occurrence may be anything from buttering a slice of bread to preparing a complex meal.

In addition to this, the sequences of several activities may overlap and also vary heavily in their occurrence depending on the habits of the operating person. Consequently, the activity recognizer has to ensure a high level of adaptability towards the basic surrounding environment and the aspired activities.

One important requirement in the context of the Ambient Assisted Living system is that the defined activity should be detected as soon as possible after its appearance. In addition, 
the chosen approach should be efficient due to the limited processing power of the hardware.

Many algorithms that deal with the detection of special patterns with and without the consideration of the time aspect are known in the field of Artificial Intelligence.

Hein and Kirste [3] have analyzed the most prominent ones in terms of their suitability in this context and have divided the approaches into Model-Free / Non-Temporal, Model-Free / Temporal, and Model-Based / Temporal approaches. They conclude that each approach has its own advantages and disadvantages, but there is no existing approach that meets all of the specific demands of activity recognition in the AAL domain as described above.

In the context of the chosen design, a few well known approaches are of special interest. One idea is to follow the timemap approach [11] for defining a typical temporal network of the sensor events that are included in the activity. The Complex Event Recognition Architecture (CERA) allows nonfuzzy recognition of special event occurrences with basic temporal dependencies. The rules are defined externally in a Lisp-like syntax [12]. Tapia et al. have chosen naive Bayesian classifiers with an extension to sequential relationships for the automatic detection of activities in elderly people's homes [13].

\section{ACTIVITY RECOGNITION FRAMEWORK}

In order to tackle the aforementioned problems, a hybrid combination of the different approaches seemed to be a promising way for us to solve most of the addressed problems in one component. Furthermore, we decided to approach the complex activities (ADLs) in a divide-and-conquer approach by decomposing them into atomic activities. Each atomic and complex activity is detected by a specialized detection agent with its own special logical unit. In order to enable arbitrary communication structures between the agents, they communicate with each other by exchanging typed facts that are represented in a common data structure.

The abstract model of the detection agent (cf. Figure 5) conforms to the idea of intelligent agents [14]. Describing the functionality of an agent in one sentence, one may say that it receives temporal shifted data, stores them in the cache memory, analyzes the data, and reports if a defined constellation has been found.

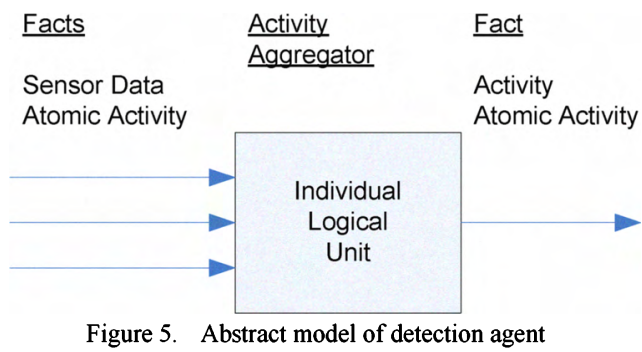

From a semantic point of view, we can classify incoming data as low-level sensor information, e.g., device usage or movement, or activities already classified by other kinds of reasoning components or by other detection agents. The number of possible incoming facts is not limited, in contrast to the outgoing activity fact, which has to be unique and is generally at a higher information level than the incoming ones. Practical experience has shown that a recursive usage can be of interest, too. This means that an agent can be interested in information that it is providing itself.

The functional characteristics of the single detection agents comply with the capabilities of software agents as defined by Wooldridge and Jennings [15], namely:

- Reactivity

- Proactiveness

- Social ability

The whole detection process is event-triggered. Primary event sources are the sensing systems and a timer, which issues periodic timer events. The different agents are activated if they receive new facts. They remember the fact with the time of its arrival and the detection algorithm proceeds if the characteristic activity pattern is present (Reactivity). In order to improve the overall performance, agents receive only those facts they are really interested in. The functionality of the reasoning algorithm is given by predefined rules; they are checked against the stored facts (Proactiveness). After the decision is made on whether the defined activity has occurred or not, the agent falls back into sleep mode. If the activity has been detected, a corresponding fact is emitted to the environment.

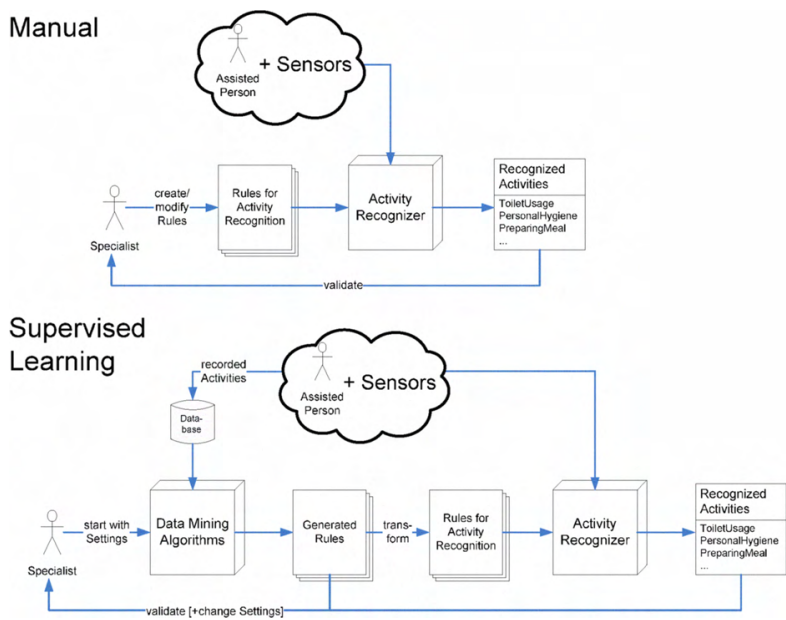

Figure 6. Configuration of the Activity Aggregator

In the first iteration of the activity recognizer, the underlying rules are defined manually by a specialist (Figure 6, top). We developed an editing tool that allows easy creation and editing of rules.

This kind of configuration entails with a few disadvantages. The overall complexity grows up very fast with the number of possible underlying events. For example, in our case we created the overall number of 92 rules for the ADLs Toilet Usage, Personal Hygiene, and Preparation of Meals. The adaptation of the rules to another instance of the system, caused by another apartment structure and maybe another sensor setting, seems to be quite complex, too. In addition to that, the adaptation of the rules to the individual behavior of the person seems to be nearly impossible without previous monitoring. 
Regarding these disadvantages of the manual configuration, a promising approach seems to be supervised learning configuration (Figure 6, bottom).

The general idea of the supervised learning configuration is that the system gathers information about the typical user's behavior during an initial learning phase with feedback from the user (alternative: camera-monitored). The cases of concrete instances of the activities of interest are stored in a central database. By using data mining algorithms, characteristic sequences are extracted. Out of these sequences, the rules for the activity recognizer are created. The configuring person (in Figure 6 called specialist) is able to change the initial setting of the data mining component afterwards to assure the best possible quality. The data mining algorithms used are described in the following chapter.

\section{REALIZATION}

In this section, we describe our realization of the approach introduced in the previous chapter. The main focus lies on the description of the recognition component. Various agents are instances of this component, with their individual logical unit described in a rule file. The recognition component and the automatic learning process are explained.

\section{A. Rules and Manual Configuration}

The logical units of the agents do not include rule definitions inside their functional code. Rules are defined in an external file in order to enable quick and easy adaptation of the rule base to specific problems. The structure of activity rules is shown in Figure 7.

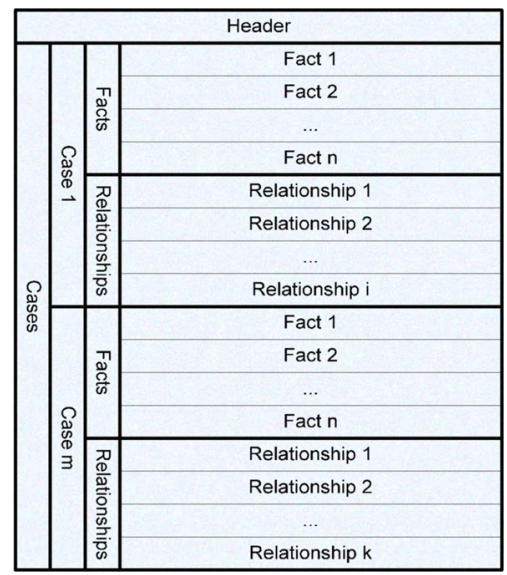

Figure 7. Structure of the activity rules

A rule header comprises declarative information about an activity, e.g., its name - which is also the name of the sent fact if the activity is detected - the maximum storage time of the cached facts, the duration of the latency period during which new received facts will be ignored after positive detection, and the threshold value that applies for every case with no individual threshold.

Furthermore, several cases of an activity can be specified. Using these different cases, different occurrences of the activity can be considered. Optionally, each case may have a specific threshold. Basically, a case consists of at least one fact, and an arbitrary number of relationships. Both types are equipped with individual characteristics.

The characteristics of a fact include a well-defined ID and name. A relationship consists of the reference to the starting fact and the ending fact, and the minimum time of occurrence between them. Facts as well as relationships have special characteristics for controlling the further flow of the activity detection process. One of these characteristics is a weight, used to support fuzzy decisions. In addition, facts or relationships can be defined as being obligatory or sufficient.

TABLE I. CHARACTERISTICS OF RULES IN AN OVERVIEW

\begin{tabular}{|l|l|}
\hline Header & $\begin{array}{l}\text { activity name, monitoring duration }(\mathrm{msec}), \\
\text { general threshold, latency duration }(\mathrm{msec})\end{array}$ \\
\hline Case & case-specific threshold (optional) \\
\hline Fact & fact ID, fact name, weight, obligatory, sufficient \\
\hline Relationship & $\begin{array}{l}\text { starting fact ID, ending fact ID, minimum in- } \\
\text { between time range (msec), weight, obligatory }\end{array}$ \\
\hline
\end{tabular}

An activity can be described by an unlimited maximum number of facts, relationships, and cases.

For supporting the configuration of the rules, an XML editor was developed (Figure 8).

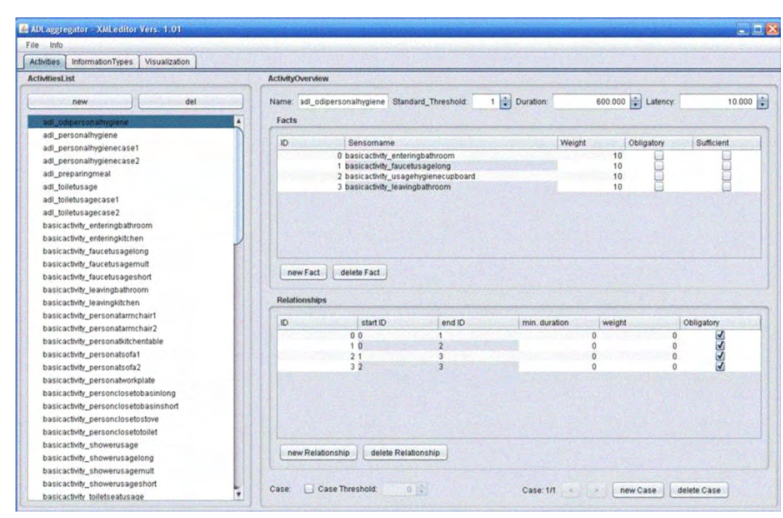

Figure 8. The XML editor supports rule configuration

\section{B. Reasoning Algorithm}

The execution of the reasoning algorithms in the agents' individual logical units is controlled by the activity rules described above. The main rationale behind this approach is that various events of interest can indicate an activity with varying weights, depending on their importance or the probability of occurrence. For example, the event toilet flush is a better indicator for the ADL Toilet Usage than light switched on in the bathroom, or motion in the bathroom.

With (weighted) facts in several self-contained cases, a fact repository for applying a case-based reasoning (CBR) approach to detect activities [16] can be set up. This way, all activities that are independent of temporal order can be described, e.g., the ADL Preparation of Meals.

Relationships between facts can extend this approach by representing the temporal orders. Thus, the accuracy of the description can be increased. 
By defining cases with at least one relationship per fact, a stringent net describing exactly the sequential temporal order of facts can be defined. Such an activity description is similar to the timemap approach or the temporal constraint networks approach, which mainly focus on the description of temporal relationships between facts [17] [18].

Facts and their relationships can be enhanced in their expressiveness by considering additional attributes. Facts or relationships with the attribute obligatory must be true for an activity to be detected. The attribute sufficient results in immediate detection of the activity.

Concerning a relationship, the attribute obligatory is only considered if both pertaining facts have occurred and the temporal constraint is fulfilled.

The definition of an overall threshold in the header or the case-specific threshold, respectively, and weights permit an individually calculable level of uncertainty. An activity is only declared as detected if the ratio of the sum of weights of all occurred facts or relationships to the sum of all weights is higher than the dedicated threshold.

\section{Multi-Agent-based Approach}

Splitting the overall reasoning approach for detecting ADLs into specialized agents that detect atomic activities makes sense for several reasons. Any kind of fact can be stored in memory once, but more instances may be of interest for the recognition of a whole activity. A simple example is the interpretation of the fact Bathroom door closed. This can argue for the subactivities Person enters Bathroom or Person leaves Bathroom as well, which are both needed for the detection of special activities in the bathroom such as the ADLs Toilet Usage or Personal Hygiene. Subactivities can be ascertained by an individual consideration that includes temporal accompanying facts like Motion in the Bathroom. Single facts can be interpreted in different ways and result in other facts at a higher level.

Another reason for the initiation of agents can be the splitting of the functionality for reasons of process optimization; only that part of the overall functionality is used that is actually affected.

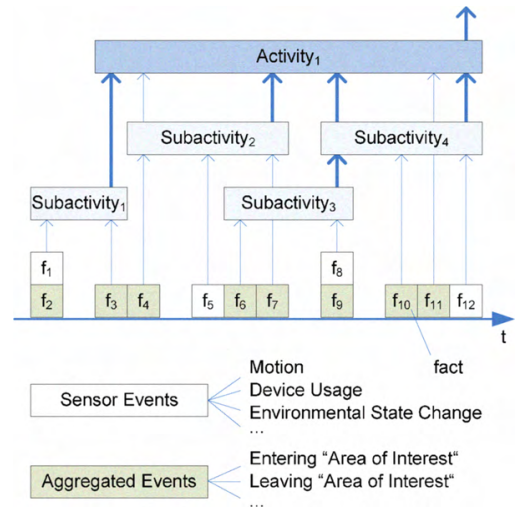

Figure 9. Exemplary activity recognition

In I.A, an example of a timeline with an on-the-fly data aggregation is illustrated. At the bottom, the occurred facts with their unique timestamps are shown. These facts may originate from sensors (motion, device usages, etc.) or already aggregated events of underlying continuous data streams, such as spatial or vital data. In the middle, specialized agents that detect subactivities depending on the constellation of facts are illustrated. The complex activity to be detected is located at the top. From the system point of view, there is no difference between subactivities and activities.

\section{Examples}

An easy example of describing the rule format is the detection of a person staying in a defined area, e.g., in front of the kitchen counter, for a longer period of time. A pressure mat could be used as a sensor.

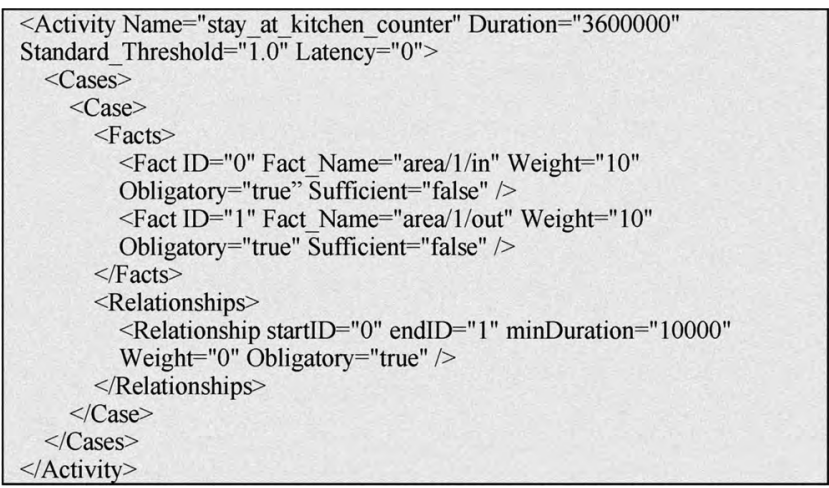

Figure 10. Example 1 (sequential): detection of stay at the kitchen_counter

In this sequential example 1, the agent detects the activity stay_in_area_l if at least ten seconds pass between the entering fact area/l/in and the leaving fact area/1/out. The maximum time for this activity is one hour $(3600000 \mathrm{msec})$.

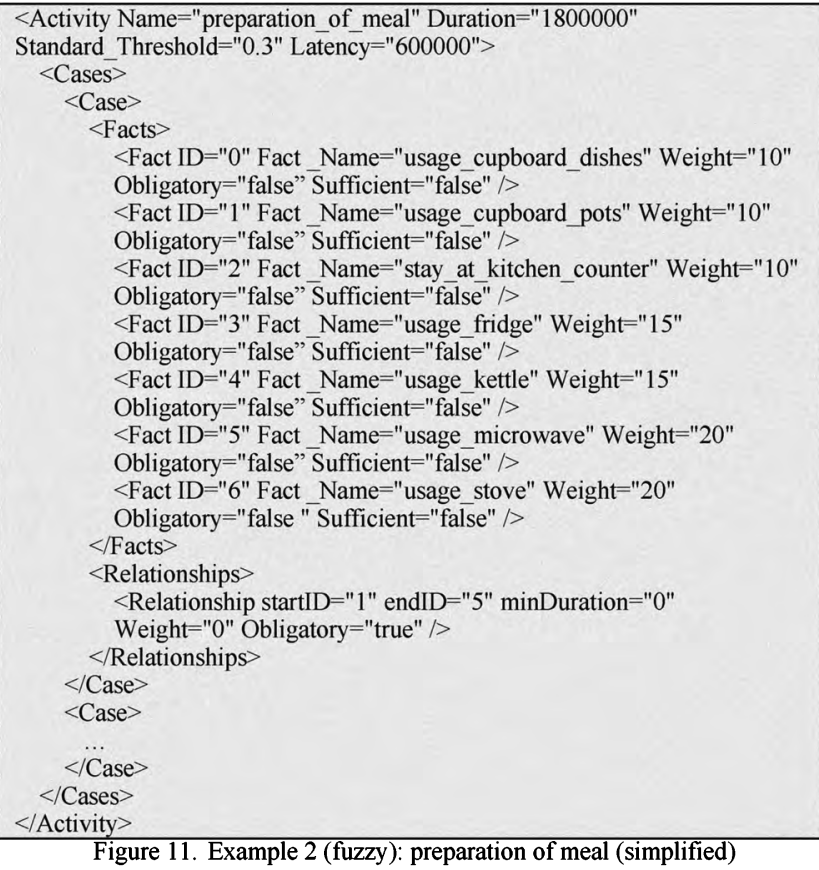

The second example demonstrates in a simplified way the usage of weights for a fuzzy reasoning, based on already 
defined (sub)activities like stay at kitchen counter (Example 1). The overall weight is 100 and the threshold, defined in the header, is 0.3 . If the sum of the weights of the occurred facts is greater than or equal to 30 , the activity is detected. One constraint is that usage_cupboard pots has to take place before usage_stove, in the case that both facts occur. It is thinkable to define more cases with different weights and thresholds.

\section{E. Automatic Generation of Rules}

The challenge of automatic rule generation is that the rules created have to be transformed into the described rule format of the activity recognizer. During the research for data mining algorithms, the subdomain of mining frequent subsequences has been of special interest. The algorithms of this subdomain find frequent subsequences of a set of sequences. The advantage of the resulting set of these algorithms is that they can be easily transferred into the rules of the activity recognizer. The disadvantage is that no good results can be achieved with frequent subsequences.

During the next steps of the research, the PrefixTWEAC algorithm was found [19], which is based on the PrefixSpan algorithm [21]. This algorithm creates characteristic subsequences on the basis of mining frequent subsequences that can be used for classification. The first step is to record several sequences for each activity that should be recognized within the learning phase. Then the algorithm finds characteristic patterns. A characteristic pattern is a frequent subsequence of the sequences of one class that does not appear in the database of other classes. Of course, one characteristic pattern cannot cover all the different kinds of an activity. This is the reason why several characteristic patterns are generated. After this step, a specialist can validate these patterns and then restart the algorithm with other parameter settings, or the rules can be transformed into the format of the activity recognizer.

The following example will easily show when a pattern is characteristic. Concerning the activity Toilet Usage, the usage of the faucet will be included in every recorded case of this action. Consequently, the sensor information of this action is a frequent pattern. Because of the fact that this pattern occurs just as often in other activities such as Personal Hygiene, this pattern is not characteristic for Toilet Usage. An extension of the pattern with the fact usage of the toilet flush will be frequent for these cases, but will not appear in the sequences of other activities. So this extended pattern is characteristic for the activity Toilet Usage.

\section{F. Practical Experiences}

The rule-based multi-agent approach described above has been implemented and integrated into the Ambient Assisted Living environment at Fraunhofer IESE. In addition to the actual functionality, a GUI for visualizing the results using a timeline (Figure 12) and an XML editor (Figure 8), which allows easy management of the agents and rules, are provided.

In an evaluation, the accuracy of the detection of ADLs was tested by randomly playing hundred scenarios with six test persons in the AAL apartment with manually defined rules. The system recognized all fifty Toilet Usage activities correctly. Two out of fifty other activities also located in the bathroom were classified incorrectly as Toilet Usage. Analogue to this experimental setting, an evaluation was done for the ADLs Personal Hygiene and Preparation of Meals. The results of all scenarios were transformed into confusion matrices. The accuracy was calculated and subsequently a $\chi^{2}$-test was used to verify the independence of the classifier. The conclusion was that our approach seems to be well suited for the on-the-fly detection of activities in the domain of Ambient Assisted Living, in general (for further information, see [21]).

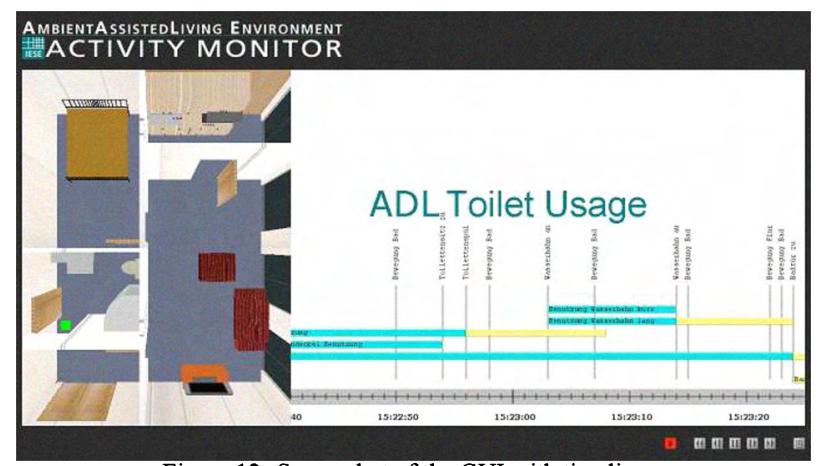

Figure 12. Screenshot of the GUI with timeline

The described reasoning component certainly has no way of distinguishing between real Toilet Usage and scenarios with a nearly identical event stream, such as Cleaning the Toilet. For long-term reasoning issues, this fact might be marginal. The general functionality of the automatic rule learning algorithm has been shown with a ten-fold cross-validation based on 75 recorded activities.

\section{CONCLUSION AND FUTURE WORK}

In this paper, we described our approach for the recognition of activities, based on the experiences we made in the domain of Ambient Assisted Living.

After describing our general motivation and giving a short introduction to the domain of AAL, we first described the theoretical view on our approach by giving examples, and second, its practical realization and the experiences we made.

In summary, it can be concluded that the approach has the potential of detecting activities, which may be of varying complexity, sequential or rather random, and overlapping with other activities as well. Regarding the sensor environment and the behavior of an elderly person, the overall recognition functionality can be adapted individually, supported by an automated learning algorithm. Incompletely received information will be considered, and incomplete knowledge can also be modeled using the given rule structure.

In terms of future work, we plan to develop an underlying ontology, including the rules for all reasoning agents. The general suitability of our approach will be verified by the configuration of more activities and advanced evaluations in the field in Greece and Germany in the context of the EMERGE project. Advanced steps include enforcing the development of the trend calculation and assessments, regarding the clinical knowledge based on ADL detection. 


\section{ACKNOWLEDGMENT}

Part of this work has been funded by the European Commission under project no. FP6-IST-2005-045056 EMERGE [2].

\section{REFERENCES}

[1] Ambient Assisted Living Joint Program, http://www.aal-europe.eu, last visited 10.04.2008

[2] EMERGE, Emergency Monitoring and Prevention, EU project, http://www.emerge-project.eu, last visited 26.02.2009.

[3] A. Hein and T. Kirste, "Activity Recognition for Ambient Assisted Living: Potential and Challenges", 1st German AAL-Congress, Berlin, 2008.

[4] H. Wilson, "Assistive Intelligent Environments for Automatic Health Monitoring", Ph.D. dissertation, Robotics Institute, Carnegie Mellon University, 2005, pp. 8.

[5] Ambient Assisted Living Environment, http://aal.iese.fraunhofer.de, last visited 26.02.2009.

[6] mst news: "Ambient Assisted Living", mstnews 06/07, http://www.mstnews.de/past-issues, last visited 26.02.2009.

[7] J. Nehmer, A. Karshmer, M. Becker, and R. Lamm, "Living Assistance Systems - An Ambient Intelligence Approach", International Conference on Software Engineering (ICSE), 2006.

[8] M. Nick and M. Becker, "A Hybrid Approach to Intelligent Living Assistance", Hybrid Intelligent Systems, Kaiserslautern, 2007, pp. 2-3.

[9] S. Katz, A.B. Ford, R.W. Moskowitz, B.A. Jackson, and M.W. Jaffe, "Studies of Illness in the Aged: The Index of ADL: A Standardized Measure of Biological and Psychosocial Function", Journal of the American Medical Association, 185(12), 1963, pp. 914 - 919

[10] H. Storf, and M. Becker, "A Multi-Agent-based Activity Recognition Approach for Ambient Assisted Living", 3rd Workshop Artificial Intelligence Techniques for Ambient Intelligence, European Conference on Artificial Intelligence, Patras, Greece 2008

[11] M. E. Pollack, "Intelligent Technology for an Aging Population - The Use of AI to Assist Elders with Cognitive Impairment", American Association for Artificial Intelligence, 2005, pp. 9-24.
[12] W. Fitzgerald, R. James Firby, A. Phillips, and J. Kairys, "Complex Event Pattern Recognition for Long-Term System Monitoring", Workshop on Interaction between Humans and Autonomous Systems over Extended Operation, San Mateo, CA, 2004.

[13] E. M. Tapia, S. S. Intille, and K. Larson, "Activity Recognition in the Home Using Simple and Ubiquitous Sensors", Pervasive Computing, Vienna, 2004, pp. 4

[14] M. Wooldridge, "An Introduction to MultiAgent Systems", John Wiley \& Sons, Chichester, 2002, pp. 16.

[15] M. Wooldridge, and N. R. Jennings, "Intelligent Agents: Theory and Practice", The Knowledge Engineering Review, 10(2), 1995, pp. 115152

[16] A. Aamodt, and E. Plaza, "Case-Based Reasoning: Foundational Issues, Methodological Variations, and System Approaches", AICom Artificial Intelligence Communications, IOS Press, Vol. 7: 1, 1994, pp 39-59.

[17] C. Dousson, P. Gaborit, and M. Ghallab, "Situation Recognition: Representation and Algorithms", 13th IJCAI, Chambéry, France, 1993 pp. 166-172.

[18] M. Dojat, N. Ramaux, and D. Fontaine, "Scenario Recognition for Temporal Reasoning in Medical Domains", Artificial Intelligence in Medicine 14, 1998, pp. 139-155.

[19] E. H. de Graaf, and W. A. Kosters, "Efficient Feature Detection for Sequence Classification in a Receptor Database", Belgian-Netherlands Conference on Artificial Intelligence (BNAIC), Brüssel, Belgien, 2005

[20] J. Pei, J. Han, B. Mortazavi-Asl, H. Pinto, Q. Chen, U. Dayal, and M.-C Hsu, "PrefixSpan: Mining Sequential Patterns Efficiently by PrefixProjected Pattern Growth", International. Conference on Data Engineering (ICDE), Heidelberg, Germany, 2001.

[21] T. Kleinberger, A. Jedlitschka, H. Storf, S. Steinbach-Nordmann, and S. Prückner, „An Approach to and Evaluations of Assisted Living Systems using Ambient Intelligence for Emergency Monitoring and Prevention", 13th International Conference on Human-Computer Interaction, San Diego, USA, 2009 (in print) 